\title{
Evaluation of shoulder complex motion-based input strategies for endpoint prosthetic-limb control using dual-task paradigm
}

\author{
Yves Losier, MScEE, PhD, PEng; ${ }^{*}$ Kevin Englehart, MScEE, PhD, PEng; Bernard Hudgins, MScEE, PhD, PEng \\ Institute of Biomedical Engineering, Department of Electrical and Computer Engineering, University of New Brunswick, \\ Fredericton, Canada
}

\begin{abstract}
This article describes the design and evaluation of two comprehensive strategies for endpoint-based control of multiarticulated powered upper-limb prostheses. One method uses residual shoulder motion position; the other solely uses myoelectric signal pattern classification. Both approaches are calibrated for individual users through a short training protocol. The control systems were assessed both quantitatively and qualitatively with use of a functional usability protocol based on a dual-task paradigm. The results revealed that the residual motion-based strategy outperformed the myoelectric signalbased scheme, while neither strategy appeared to significantly increase the mental burden demanded of the users.
\end{abstract}

Key words: amputation, dual-task paradigm, EMG, endpoint control, myoelectric signals, pattern recognition, powered prostheses, rehabilitation, residual limb position, shoulder motion.

\section{INTRODUCTION}

Significant research has occurred in the past several decades to achieve suitable solutions for the control of prosthetic upper limbs [1]. Although the development of advanced and sophisticated strategies has progressed steadily for more distal amputation cases, very limited improvements have occurred for more proximal amputation levels. This is due, in part, to the fact that the majority of cases are below-elbow amputations while higherlevel amputations, such as shoulder disarticulation, are less common [2]. The functional requirement of the prosthesis increases with the level of amputation, which leads to a paradox for the control strategy because the number of available input sources decreases. As a result, the need for a robust and intuitive strategy is most critical for high-level amputation cases in order to regain some acceptable level of function with the artificial limb.

Under ideal conditions, a synergistic relationship could be developed between the neural activity of the person with an amputation and the associated missing degrees of freedom (DOFs). Invasive approaches, such as cortical [3] or peripheral nerve [4] interfaces, show promise but are years away from practical application. However, noninvasive alternatives have existed for several decades. These alternatives include monitoring of either the movement of a residual limb (or of another part of the body) or the electrical activity accompanying voluntary contraction of one or multiple muscles. A mode switch is often required to select amongst the requisite DOFs if the number of control sources is less than the number of DOFs to be controlled.

The most common form of input source and control scheme for high-level upper-limb amputation is the use of residual movements to drive cable-operated joints. This body-powered method has been in use for many decades and is currently the most clinically available option. Its

\footnotetext{
Abbreviations: $\mathrm{DOF}=$ degree of freedom, $\mathrm{LDA}=$ linear discriminant analysis, MES = myoelectric signal, TD = time domain.

*Address all correspondence to Yves Losier, MScEE, PhD, PEng; Institute of Biomedical Engineering, Department of Electrical and Computer Engineering, University of New Brunswick, PO Box 4400, Fredericton, NB E3B 5A3, Canada; 506-458-7026; fax: 506-453-4827. Email: ylosier@unb.ca DOI:10.1682/JRRD.2010.08.0165
} 
importance is amplified for high-level amputation cases where the availability of robust input control sources is often limited. Externally powered systems do exist in which sensors, such as force-sensing resistors, joysticks, linear transducers, and rocker switches, are activated by the user's residual motion. However, these can prove to be cumbersome, nonintuitive means of control.

Control of powered devices with use of the myoelectric signal (MES) originating from the residual limb and shoulder complex has had limited success [5-7]. Conventional (or direct) control uses the amplitude of one or two muscles to control each DOF and requires some mode switch to select amongst multiple DOFs. Alternatively, pattern classification schemes have been used with the MES in an attempt to capture the complex synergy in the shoulder musculature and map this to multiple DOFs. Buerkle used a linear discriminant analysis (LDA) algorithm to classify eight shoulder motions corresponding to fundamental anatomical DOFs (shoulder flexion/extension, medial/lateral rotation, abduction, adduction, and transverse flexion/extension) [8]. The hypothesis was that the ability to classify natural shoulder contractions from the remaining musculature would provide an intuitive means of controlling multiple DOFs at the shoulder. This study demonstrated that these motions could be classified with high accuracy but users had considerable difficulty when performing functional tasks requiring the use of multiple DOFs. This is likely because reaching and positioning tasks require the execution of multiple anatomical DOFs, either simultaneously or in sequence. Simultaneous control of multiple DOFs with use of an LDA scheme is not yet possible, and sequential control was nonintuitive for users and resulted in a considerable mental burden during the experiment.

The clinical team's selection of a control strategy will depend heavily on the consideration of several design factors (patient's musculature condition, range of motion, learning ability, etc.) in order to obtain an appropriate prosthetic rehabilitation plan [7]. Other design issues, such as sensor orientation and output range, also require some consideration before the fabrication of the prosthesis because some level of final adjustments and modifications will invariably be required with any devised solution. Ideally, it would be beneficial to have an initialization protocol by which some of these factors would be considered and their associated implementation complexity removed from the prosthetic-limb design stages. Automatic tailoring of the system for factors such as the user's range of motion, the sensor type, positioning, and output range would speed up the clinical fitting process.
Devising alternative methods of using shoulder motion to produce robust input sources will be necessary for intuitive and natural control of a prosthetic limb. Previous research has illustrated users' ability to manipulate a prosthetic device by using an endpoint control system [9-13]. Unfortunately, these systems lacked the ability to be automatically optimized based on the characteristics of the user and prosthetic limb. The inclusion of such a feature could have resulted in some of the reported implementations being made into clinically viable solutions.

This work describes two new self-adjusting input strategies for the endpoint control of a prosthetic system in high-level amputation cases. Qualitative and quantitative measures, used to investigate the strategies' efficacy and associated mental burden, are developed and presented.

\section{METHODS}

Two endpoint control schemes were investigated in this work. Two novel methods of determining shoulder motions were developed:

1. An MES classification scheme that classifies physiologically intuitive shoulder motions.

\section{A robust mapping of residual shoulder motion.}

These methods provided the intended shoulder motion; to realize an endpoint control scheme, we iteratively resolved the shoulder, humeral, and elbow angles by using an inverse kinematic solution. This required that a Jacobian inverse be calculated and used with the endpoint position, whose value is continuously modified by the intended shoulder motion output values.

\section{MES-Based Classification Strategy}

Simple contractions, which were deemed to be intuitive and physically achievable for people with high-level amputations, were chosen to improve the chances of developing a practical MES-based classification strategy. Unlike previous work that only used contraction relating to humeral movements [14], the MES strategy used contractions elicited during performance of more physiologically relevant shoulder-girdle movements. The four different discrete shoulder-girdle movements selected were elevation, protraction, depression, and retraction. In addition, the muscle contractions related to both internal and external humeral rotations were included in the motion set, along with a nomovement class. 
An LDA classification scheme was chosen, given that previous research has shown it capable of providing a simple yet robust MES-based pattern-recognition strategy for the control of prosthetic limbs [15]. A feature set consisting of time domain (TD) statistics, used previously in real-time MES control schemes [15-17], was also selected for the MES-based strategy. Included in the TD set are the number of zero crossings, the waveform length, the number of slope sign changes, and the mean absolute value for a given data window.

\section{Shoulder Position-Based Strategy}

A shoulder position-based input strategy, termed "vector projection," was developed to adapt to several user and input sensor variables based on a short data collection protocol performed before use of the prosthetic limb [18]. The vector-projection algorithm addresses the issue of automatic system calibration by transforming the input sensor signals based on residual shoulder-girdle motion data collected during a short training session. The training motions provide the necessary information for calculation of a state centroid and vector for each of the corresponding motions within the input signal space (Figure 1).

These state vectors are computed for each desired motion. Note that the range of motion and absolute orientation for each state have been incorporated into the magnitude of its associated vector. This results in the removal of dependencies associated with the input sensor type,

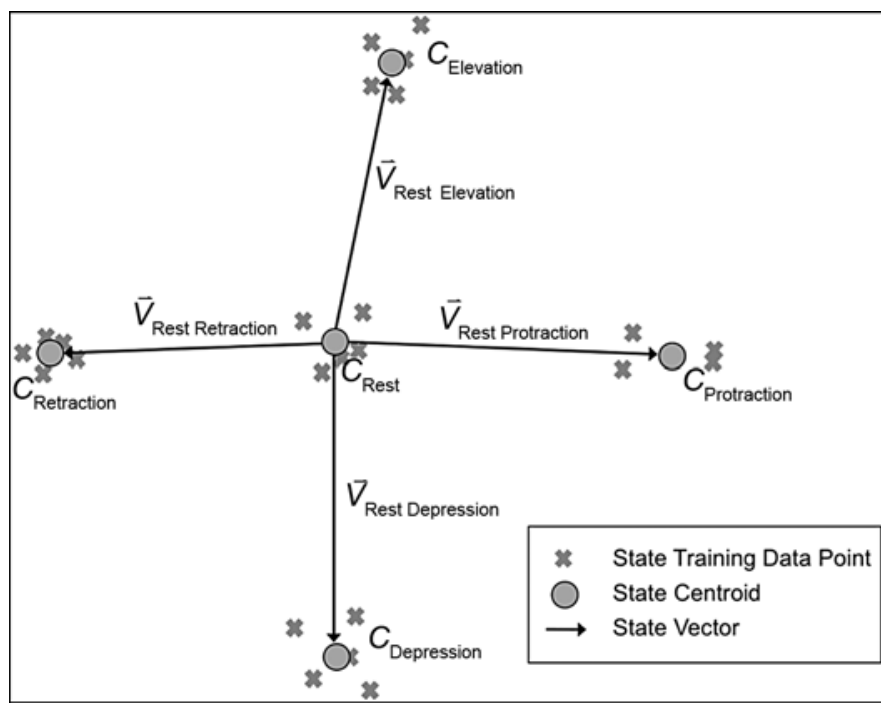

Figure 1.

State vector diagram for vector-projection algorithm. $C=$ centroid, $\vec{V}=$ vector. orientation, range, and alignment chosen by the clinical fitting team.

The implementation of real-time control involves computing a vector of the current shoulder position (OCurrent) with respect to the neutral (rest) state (ORest) (Equation 1):

$$
\vec{V}_{\text {RestCurrent }}=\vec{V}_{\text {OCurrent }}-\vec{V}_{\text {ORest }} .
$$

The magnitude of this vector and the angles between it and the adjacent state vectors can be computed with standard geometry. These vectors can also be projected onto any of the state vectors (Figure 2); the projected result represents the normalized input vector magnitude for the given state $x$ (Equation 2):

$$
d_{x}=\frac{\left|\vec{V}_{\text {Rest Current }}\right|}{\left|\vec{V}_{\text {Rest } x}\right|} .
$$

Two tunable coefficients for each state vector, with values ranging from 0 to 1 , allow a clinician to further tailor the output signals while fitting the prosthetic limb. The first coefficient, termed "magnitude coefficient," sets a region of no activity near the rest-state centroid such

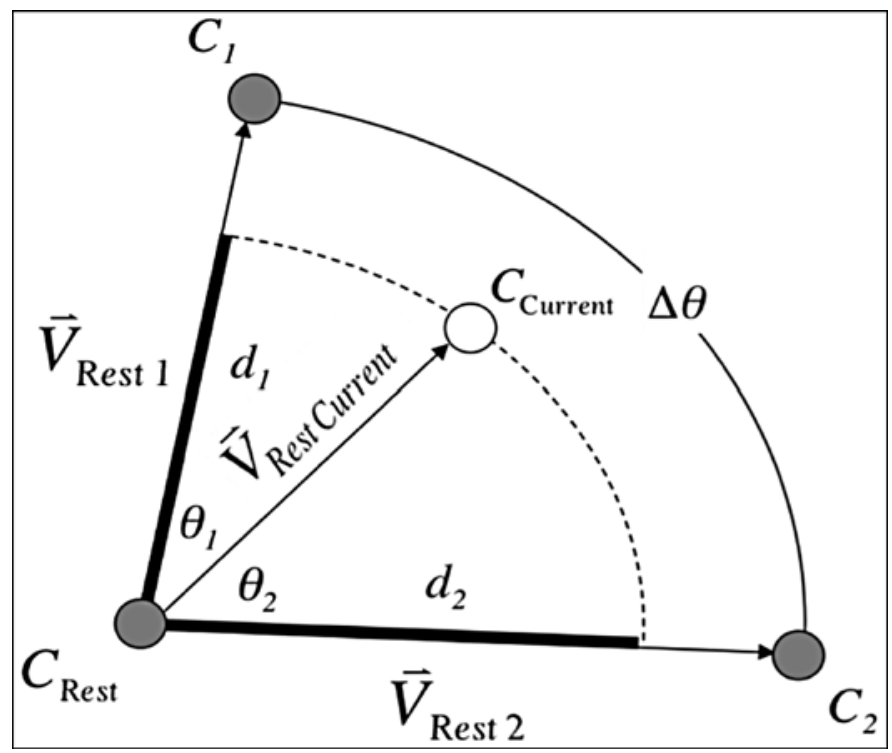

Figure 2.

Simultaneous projection of input vector onto adjacent class vectors. $C=$ centroid; $d=$ normalized magnitude; $\theta=$ angle between current input and adjacent state vectors, normalized magnitude; $\vec{V}=$ vector. 
that no inadvertent output activations occur. The second coefficient, termed "offset coefficient," reduces the effective output signal amplitude as the angle between the input and state vectors increases. These coefficients provide the information necessary to transform the original input sensor values into more meaningful control-system inputs with increased robustness (Figures 3-4). As can be seen in Figure 3, the use of a small magnitude coefficient will reduce the range of the inactive rest state. Using a larger magnitude coefficient, as seen in Figure 4, increases the necessary shoulder displacement required to generate an output signal for the given state (elevation in this case). This necessitated increase in shoulder displacement may be desirable to ensure that unintentional activations do not occur as a result of minor displacements when the user is at rest. Also, the use of a large offset coefficient (Figure 3) will minimize the weakening effect imposed on the output signal's amplitude as the angle between the input and state vectors increases. Using a smaller offset coefficient value (Figure 4) allows for a smaller active area, which may or may not be desirable depending on the user's range of motion and, more importantly, ability to reproduce the desired movement.

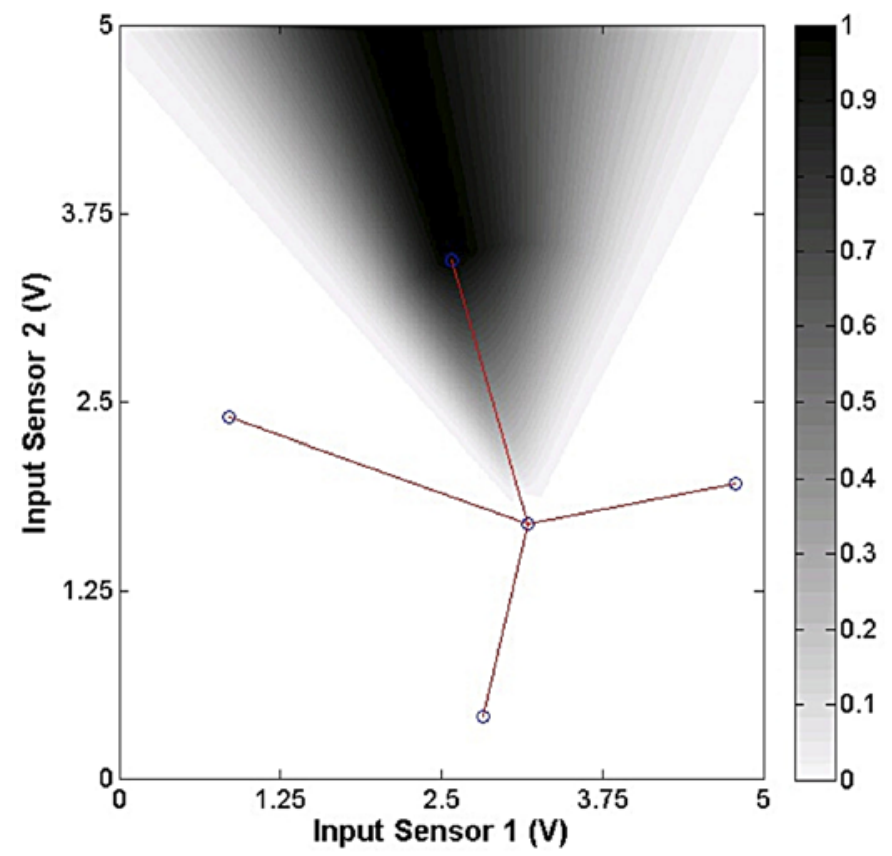

Figure 3.

Elevation state output strength when magnitude and offset coefficients are set to 0.1 and 0.5 , respectively.

\section{Dual-Task Functional Testing}

We devised a dual-task paradigm to provide possible insight into how much effort and concentration the user would require to effectively control the endpoint of a prosthetic limb using one of the two described input strategies. This is accomplished by periodically requiring a user to perform a secondary task concurrently with a primary task [19].

The primary task consisted of a three-dimensional reaching task within the prosthetic device's workspace. Protraction and retraction motions controlled the forward and backward movements of the manipulator's endpoint. Elevation and depression motions were mapped to the upward and downward motions. Finally, the internal and external humeral rotations controlled the left and right movements. In the case of the shoulder position-based strategy, an MES-based classifier, based on internal and external humeral rotation, provided the additional input sources required to operate the usability test's endpoint control scheme. Note, however, that such a classifier could be replaced by other means of measuring the humeral rotation [20] as they become available.

The secondary task consisted of a reaction assessment task and captured a measure of the mental burden associated

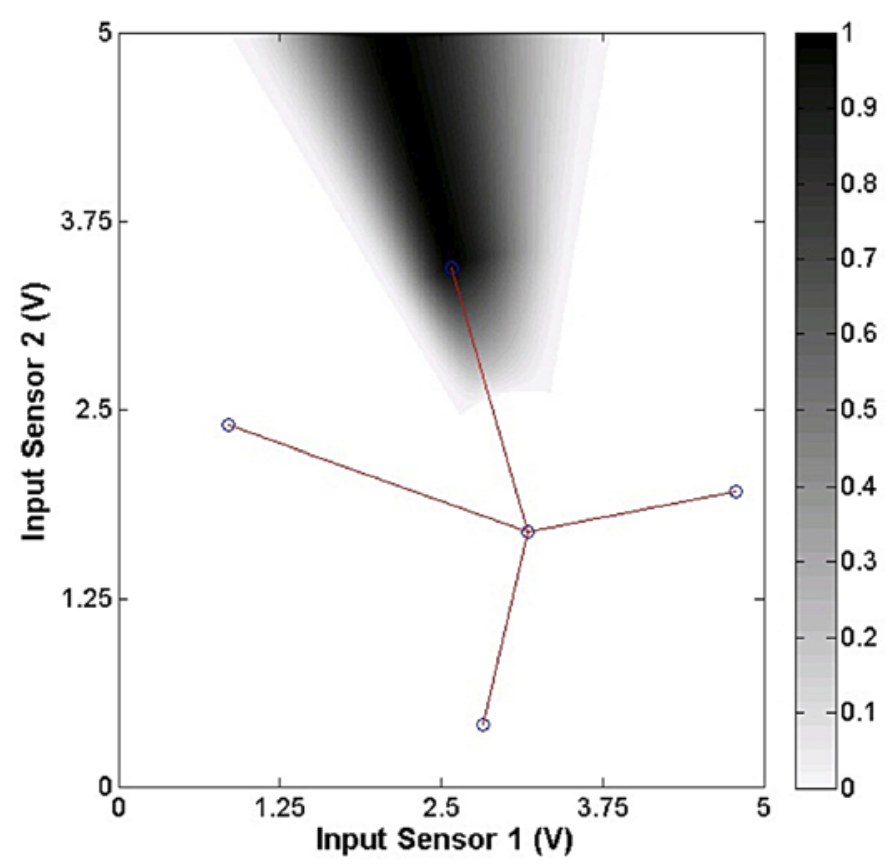

Figure 4.

Elevation state output strength when magnitude and offset coefficients are set to 0.5 and 0.3 , respectively. 
with the primary task. An experimental apparatus was designed and fabricated with the intended purpose of-

- Acquiring both shoulder position and MES originating from the shoulder complex.

- Providing a manipulator device capable of handling endpoint control.

- Quantitatively measuring the input strategy performance for gross movement tasks.

- Quantitatively capturing some measure of mental burden.

\section{Primary Task: Usability Test}

The goal of the usability test was to evaluate each input strategy's ability to control the position of an end effector during a reaching task. As a result, we preferred to solely concentrate on evaluating the gross movement of the manipulator rather than focusing on fine manipulation displacement, because these would normally be linked to the DOFs of a prosthetic limb's hand and wrist components. The inverse kinematics algorithm required to calculate the desired endpoint position uses the device's current joint angles to successfully converge to the new joint angle values. Given that no commercially available prosthesis provides the necessary positional feedback for such an implementation, a servo-based manipulator was designed and used for the experiment (Figure 5). The control strategy's output signals were transmitted to the manipulator embedded control system to actuate its servomotors.

Users were required to press target buttons by controlling the manipulator using one of the input strategies.

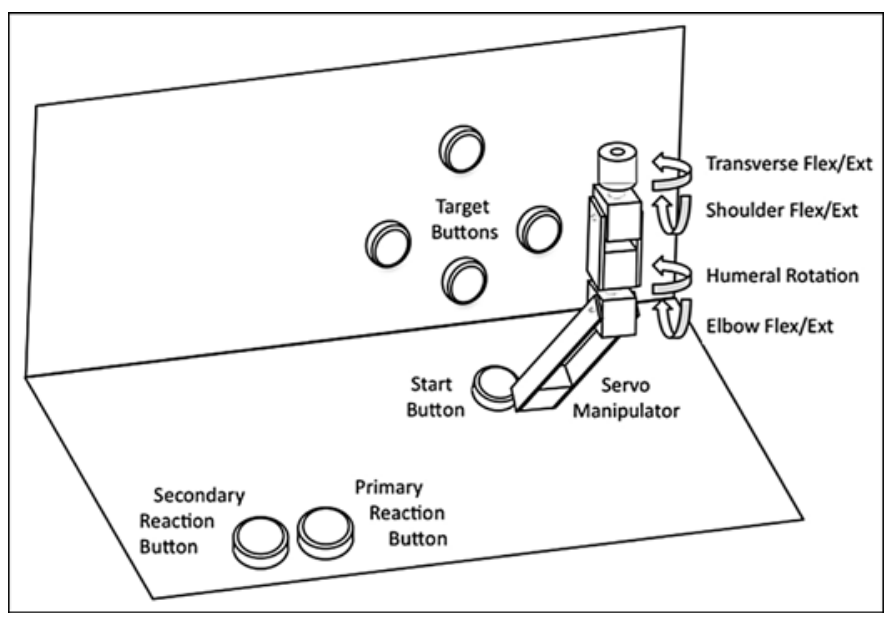

Figure 5.

Experimental apparatus setup. Flex/Ext = flexion/extension.
A trial consisted of moving the manipulator to (1) press the illuminated start button, (2) press the randomly selected illuminated target button, and (3) repeat the process until instructed otherwise.

\section{Secondary Task: Reaction Test}

The purpose of the reaction test was to periodically introduce a secondary task in the experiment that allowed for quantitative measurement of the user's mental burden. It included two push buttons and a buzzer that would instruct the user when to perform the secondary task. The users were required to rest their left hand by pressing the nearest reaction button located in front of them. During the course of the trial, the buzzer would activate, which indicated to the users to release the currently pressed reaction button, press the secondary reaction button, and return their hand back to a resting position by re-pressing the primary reaction button.

The reaction test was not activated for every target used in the primary task. The prompting of the secondary reaction task was randomized in an attempt to avoid any user anticipation during the course of the experiment. A random time delay (varying between 0 and 2 seconds) was also added before the start of the secondary task to further reduce the user's ability to anticipate the commencement of the reaction test. The mental-burden metric used for the data analysis is expressed as the difference between the time $(T)$ to depress the primary button when performing both the reaction and usability tests and the time to depress the same button when solely performing the reaction test (Equation 3):

$$
\Delta_{\text {Reaction }}=T_{\text {Reaction During Usability Test }}-T_{\text {Reaction Only }}
$$

\section{EXPERIMENTAL PROCEDURE}

The experiment consisted of one session during which subjects were fitted with eight silver-silver chloride Duotrode electrodes (6140, Myotronics; Kent, Washington) placed at physiologically relevant locations (Figure 6) for the desired shoulder-girdle movements. A reference electrode (2259, 3M Health Care RedDot; St. Paul, Minnesota) was placed on the clavicular bone region midway between the sternum and acromion. A second similar electrode was placed on the acromion bone landmark and was used to attach the joystick connector (Figure 7). Note that previous research has shown that the longitudinal place- 


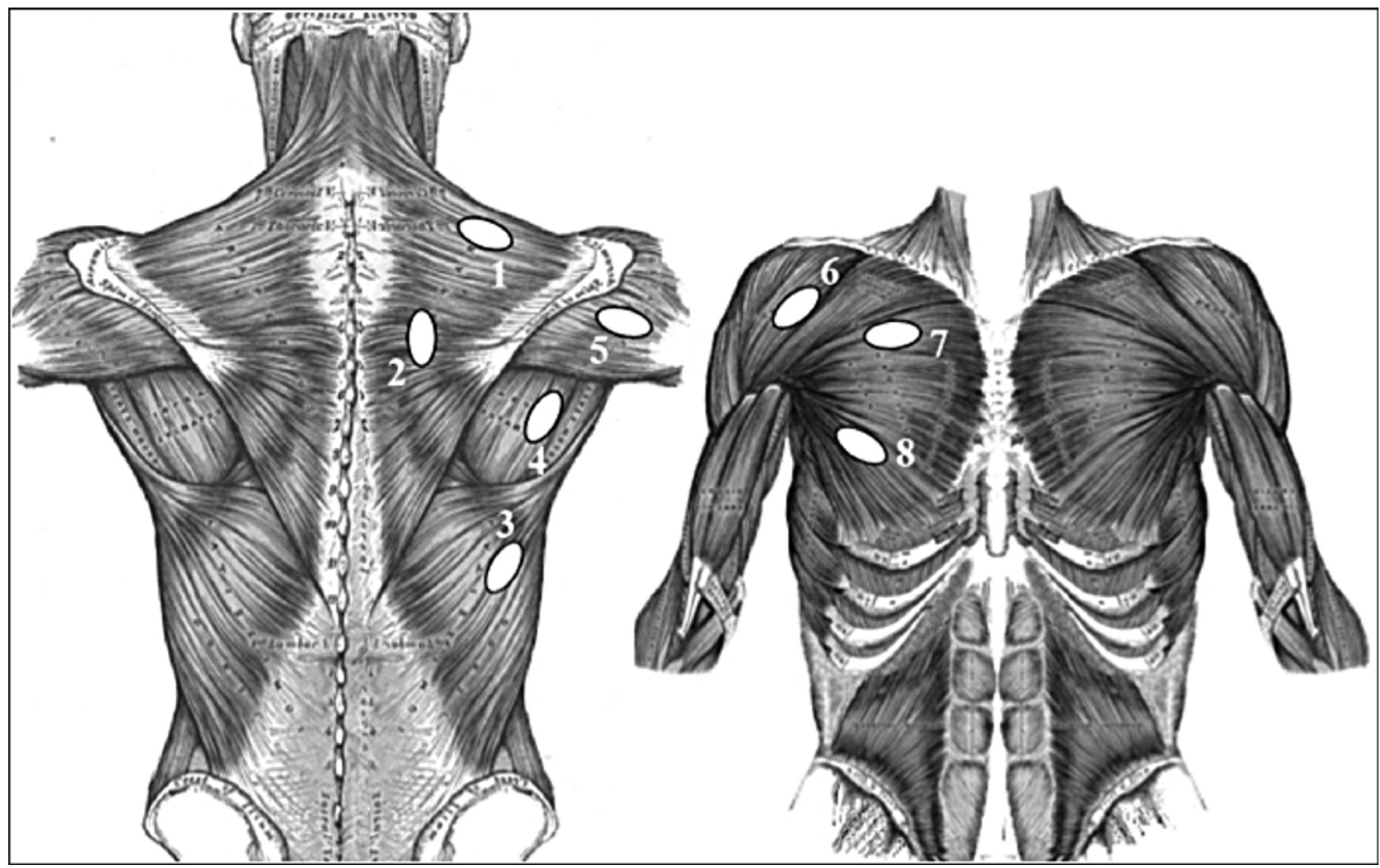

Figure 6.

Electrode (1-8) placement diagram. Source: Gray H. Anatomy of the human body. Philadelphia (PA): Lea \& Febiger; 1918. p. 409, 433, 436, 438.

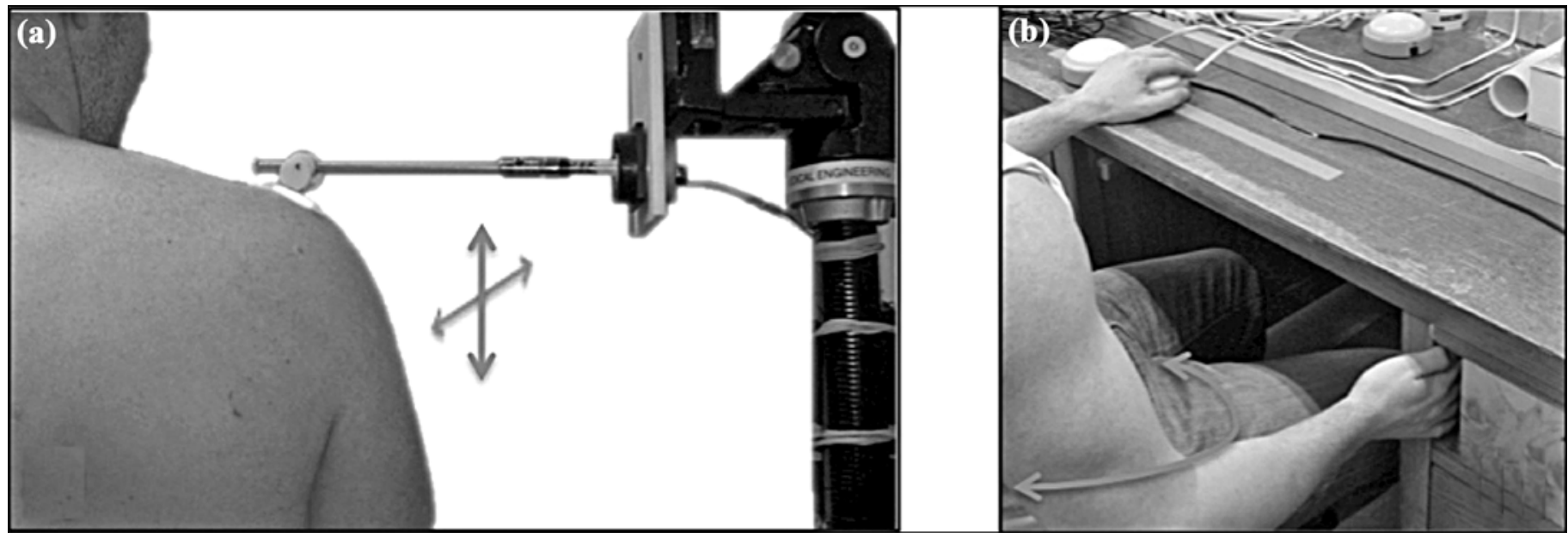

Figure 7.

Experimental setup: (a) Joystick apparatus used to capture movements used by shoulder position-based strategy and (b) subject setup for humeral rotation isometric contractions. 
ment of electrodes does not improve classification accuracy over transverse placement [21]. As a result, one electrode was positioned transversely over the rhomboid region to maximize its pickup area.

Twelve nondisabled, healthy, male individuals ranging in age from 25 to 33 were recruited to participate in this experiment. Each subject was required to provide informed consent before participating in the experiment. The University of New Brunswick Research Ethics Board approved the experimental procedure used for this research. Each subject was given a general overview of the purpose of the experiment. The data collection process was also described in detail since most subjects had no prior experience with MES data collection. The data collection protocol used for each strategy has previously been reported in literature [18,22]. The testing of a given input strategy was performed immediately following its associated training data collection session. The partici- pants practiced moving the manipulator for 5 minutes with the input strategy. The order in which the input strategies were presented was randomized to negate any learning effects associated with the experiment. Three trials were completed for each input strategy.

\section{RESULTS}

The principal objective of this experimental test was to obtain quantitative and qualitative outcomes from the input strategies under consideration. Figure 8 shows the averaged usability test outcomes for each subject, where time refers to the average time required to reach the buttons during the usability test. The data were subjected to a one-way analysis of variance to investigate statistical significance. A $p$-value of less than 0.001 was found,

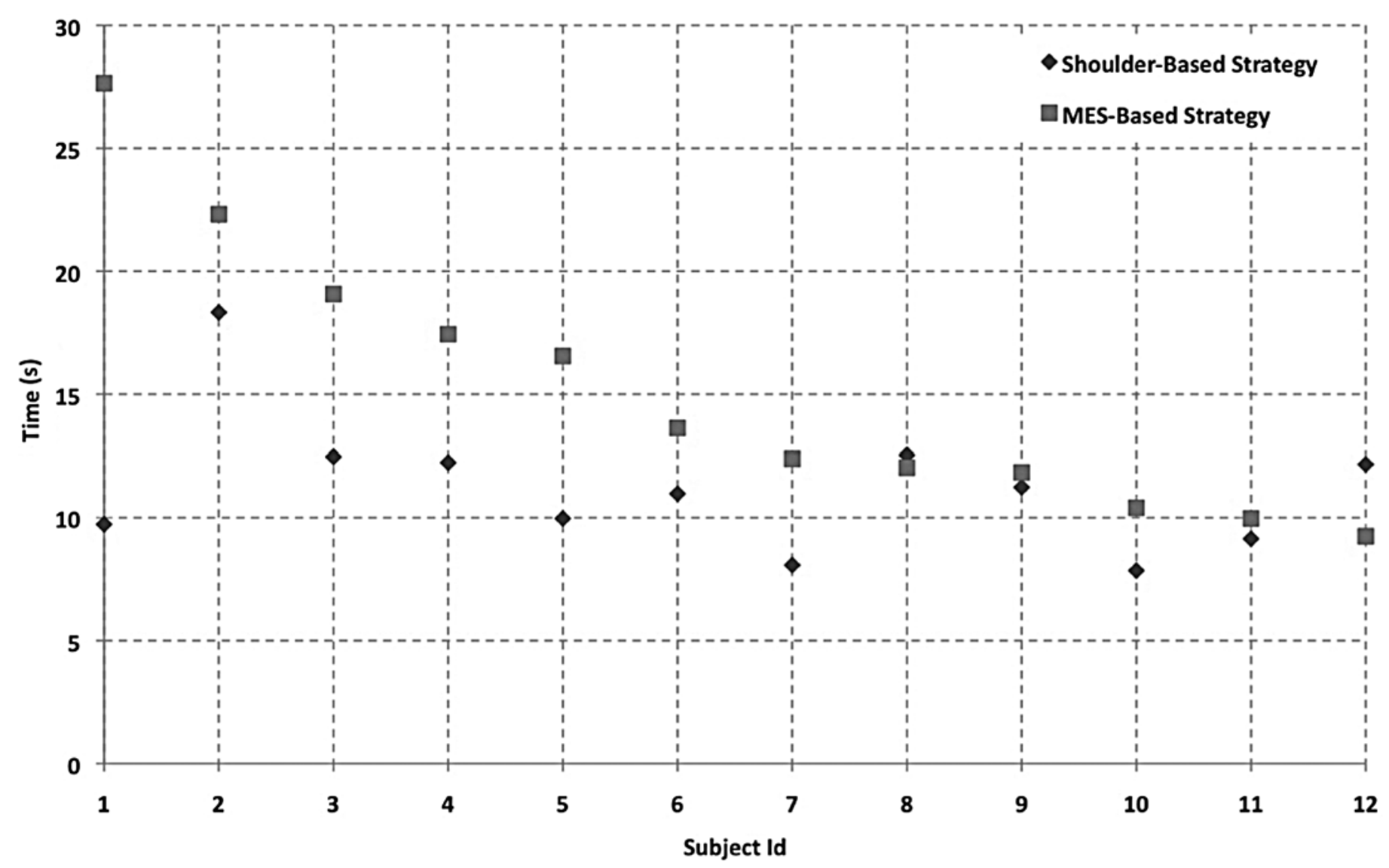

Figure 8.

Usability performance of input strategies for each subject. Subject identifiers (Ids) were renumbered to provide monotonically decreasing data curve. MES = myoelectric signal. 
indicating that the vector-projection input strategy was significantly better than the MES-based strategy.

The purpose of evaluating the reaction test outcomes was to measure the additional mental load associated with performance of the functional test. Previous research has used secondary tasks as a means of increasing the mental burden during performance of a usability task [13,23-24]. Using this method provided a measure of the mental load associated with the primary task. The experimental results (Figure 9) did not reveal any significant difference between the two strategies $(p=0.29)$. The addition of the secondary task did not significantly affect the results from the usability test.

Qualitative results, obtained through a short series of questions to the user, were also recorded upon completion of the experiment. Users were asked their preferred input strategy after completing the entire experiment. The user group mainly favored the shoulder position-based strategy (83\%) over the MES-based classifier input strategy. Users also reported fewer nonelicited manipulator actuations with the shoulder position-based strategy. These findings corroborate the experiment's quantitative results.

\section{DISCUSSION}

This research demonstrated that various input sources could be exploited to produce effective adapting strategies that provide a means of automatically calibrating and optimizing the signals used in the control scheme of a prosthetic-limb system. Although using a servo-based manipulator to measure and quantify both usability and

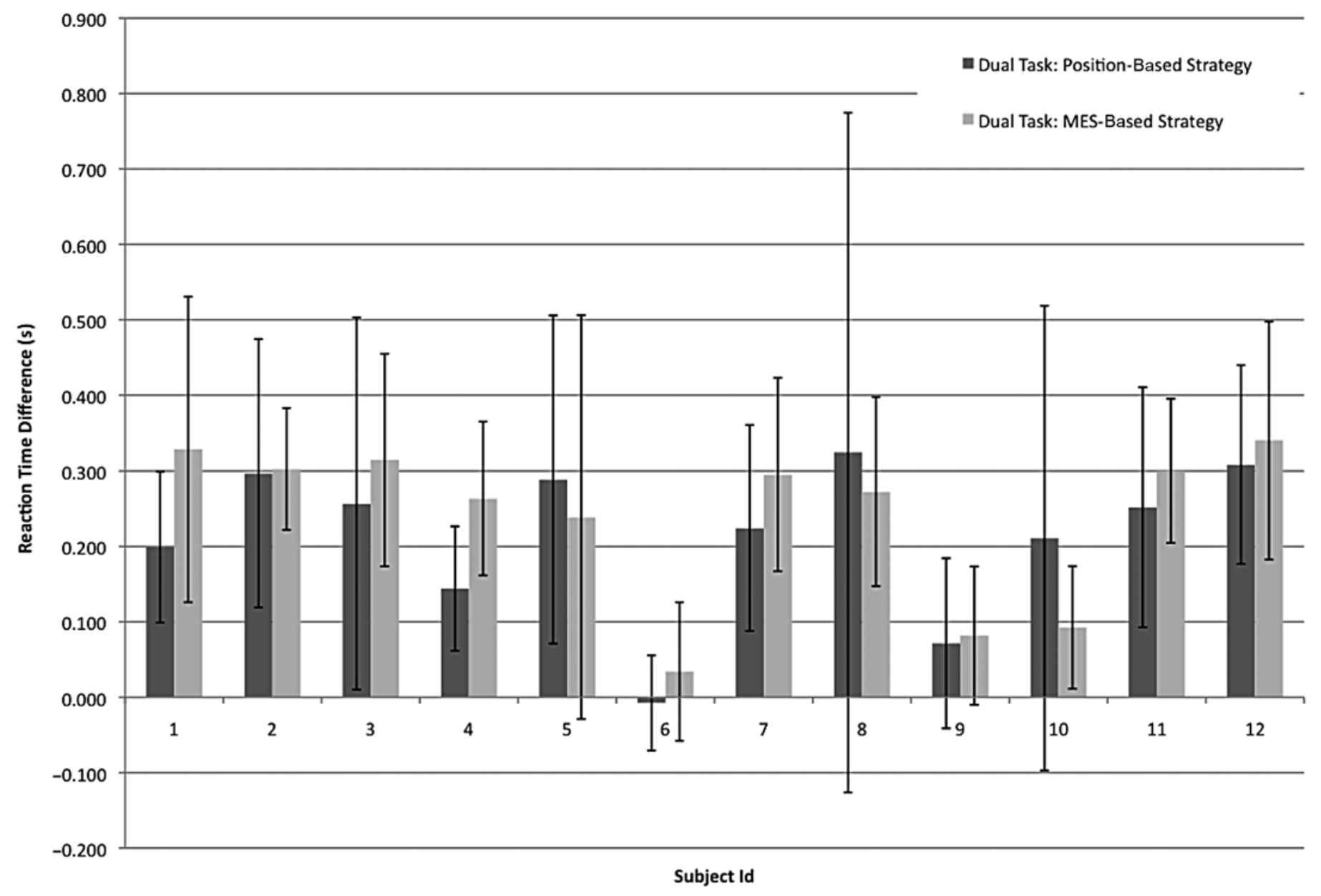

Figure 9.

Mental burden of input strategies for each subject. Subject identifiers (Ids) correspond to same order as Figure 8. MES = myoelectric signal. 
mental burden metrics is difficult, the experiment tested the usability of the system to reach fixed endpoint positions. It highlighted the ability of both strategies to use an endpoint control scheme without increasing the mental burden of the user. The strategies also facilitated the intuitive mapping of the user's residual movement with the functional space in which a prosthesis can operate.

Ongoing work has focused on the implementation of the shoulder position-based algorithm on an embedded microcontroller. Preliminary results have shown the embedded prototype to be compatible with commercially available prosthetic devices. The device is restricted to traditional control paradigms given that commercially available prostheses do not provide the necessary position feedback required for an endpoint control strategy. Despite this limitation, the automatic tailoring nature of the system has made it a viable option during the clinical fitting process.

\section{CONCLUSIONS}

This research exploited the multiple input sources found at the shoulder complex for the control of prosthetic limbs with high-level amputation cases. Both presented strategies were shown to provide an intuitive and robust means of control and have great potential for practical implementation. The investigation of the functional usability of the control strategies revealed that the shoulder position-based method outperformed the MES-based scheme, while the reaction tests showed that both strategies added little increase to the mental burden imposed on the user. A preliminary fitting has been done with a shoulder disarticulation client using an Otto Bock prosthesis, with very encouraging results. Ongoing work is focused on the simplification and standardization of the vector-projection approach so that it may be easily implemented into any system and thereby accessible to the prosthetic rehabilitation strategy of suitable candidates.

\section{ACKNOWLEDGMENTS}

\section{Author Contributions:}

Study concept and design: Y. Losier, K. Englehart, B. Hudgins. Acquisition of data: Y. Losier.

Analysis and interpretation of data: Y. Losier.

Drafting of manuscript: Y. Losier, K. Englehart, B. Hudgins. Critical revision of manuscript for important intellectual content: Y. Losier, K. Englehart, B. Hudgins.

Statistical analysis: Y. Losier.
Obtained funding: K. Englehart, B. Hudgins.

Study supervision: K. Englehart, B. Hudgins.

Financial Disclosures: The authors have declared that no competing interests exist.

Funding/Support: This material was based on work supported by the Natural Sciences and Engineering Research Council of Canada (Discovery Grants 171368-03 and 217354-01), The Research Assistantship Initiative of the New Brunswick Innovation Foundation, and the Atlantic Innovation Fund.

Institutional Review: Each subject was required to provide informed consent before participating in the experiment. The University of New Brunswick Research Ethics Board approved the experimental procedure used for this research.

Participant Follow-Up: The authors plan to inform participants of the publication of this study. The consent form signed by the participants stipulated that, if desired, they would be informed of any publication relating to the study. Participants who expressed such interest will be informed.

\section{REFERENCES}

1. Parker P, Englehart K, Hudgins B. Myoelectric signal processing for control of powered limb prostheses. J Electromyogr Kinesiol. 2006;16(6):541-48. [PMID: 17045489] DOI:10.1016/j.jelekin.2006.08.006

2. Weed R, Atkins DJ. Return to work issues for the upper extremity amputee. In: Meier RH, Atkins DJ, editors. Functional restoration of adults and children with upper extremity amputation. New York (NY): Demos Medical Publishing; 2004. p. 337-52.

3. Taylor DM, Tillery SI, Schwartz AB. Direct cortical control of 3D neuroprosthetic devices. Science. 2002;296(5574): 1829-32. [PMID: 12052948]

DOI:10.1126/science.1070291

4. Zhou R, Jiang N, Englehart K, Parker P. A computational model and simulation study of the efferent activity in the brachial nerves during voluntary motor intent. Med Biol Eng Comput. 2010;48(1):67-77. [PMID: 19937394]

5. Lake C, Miguelez JM. Evolution of microprocessor based control systems in upper extremity prosthetics. Technol Disabil. 2003;15(2):63-71.

6. Lake C, Miguelez JM. Comparative analysis of microprocessors in upper limb prosthetics. J Prosthet Orthot. 2003;15(2): 48-65. DOI:10.1097/00008526-200304000-00004

7. Miguelez JM. Critical factors in electrically powered upper-extremity prosthetics. J Prosthet Orthot. 2002;14(1): 36-38. DOI:10.1097/00008526-200203000-00010

8. Buerkle VR. Advanced pattern recognition concepts for an artificial shoulder [master's thesis]. [Fredericton (Canada)]: University of New Brunswick; 2006. 115 p.

9. Doubler JA, Childress DS. An analysis of extended physiological proprioception as a prosthesis-control technique. J Rehabil Res Dev. 1984;21(1):5-18. [PMID: 6527290] 
10. Simpson DC, Kenworthy G. The design of a complete arm prosthesis. Biomed Eng. 1973;8(2):56-59. [PMID: 4691519]

11. Storey N. Control of an arm prosthesis [dissertation]. [Southampton (UK)]: University of Southampton; 1977.

12. Au AT, Kirsch RF. EMG-based prediction of shoulder and elbow kinematics in able-bodied and spinal cord injured individuals. IEEE Trans Rehabil Eng. 2000;8(4):471-80.

[PMID: 11204038$]$ DOI:10.1109/86.895950

13. Jones B, Kyberd P. Improved control for an artificial arm. Proceedings of the MEC'05 Myoelectric Controls Symposium; 2005 Aug 17-19; Fredericton, Canada. p. 112-16. Available from: http://openprosthetics.org/literature/91/mec05.

14. Buerkle VR, Englehart K, Hudgins B. Pattern recognition of single and combined motions from the shoulder complex. Proceedings of the 28th Annual International Conference of the IEEE Engineering in Medicine and Biology Society; 2006 Aug 30-Sep 3; New York City, NY. p. 3419-22.

15. Englehart K, Hudgins B. A robust, real-time control scheme for multifunction myoelectric control. IEEE Trans Biomed Eng. 2003;50(7):848-54. [PMID: 12848352] DOI:10.1109/TBME.2003.813539

16. Hudgins B, Parker P, Scott RN. A new strategy for multifunction myoelectric control. IEEE Trans Biomed Eng. 1993; 40(1):82-94. [PMID: 8468080] DOI:10.1109/10.204774

17. Huang Y, Englehart KB, Hudgins B, Chan AD. A Gaussian mixture model based classification scheme for myoelectric control of powered upper limb prostheses. IEEE Trans Biomed Eng. 2005;52(11):1801-11. [PMID: 16285383] DOI:10.1109/TBME.2005.856295

18. Losier Y, Englehart K, Hudgins B. Residual shoulder motion vector projection. Proceedings of the 31st Canadian Medical and Biological Engineering Society Conference; 2008 Jun 11-13; Montreal, Canada.

19. Pashler H. Dual-task interference in simple tasks: Data and theory. Psychol Bull. 1994;116(2):220-44. [PMID: 7972591] DOI:10.1037/0033-2909.116.2.220
20. Li G, Kuiken TA. Modeling of prosthetic limb rotation control by sensing rotation of residual arm bone. IEEE Trans Biomed Eng. 2008;55(9):2134-42. [PMID: 18713682] DOI:10.1109/TBME.2008.923914

21. Kang WJ, Shiu JR, Cheng CK, Lai JS, Tsao HW, Kuo TS. The effect of electrode arrangement on spectral distance measures for discrimination of EMG signals. IEEE Trans Biomed Eng. 1997;44(10):1020-23. [PMID: 9311170] DOI:10.1109/10.634653

22. Losier Y, Englehart K, Hudgins B. Residual shoulder motion MES classifier. Proceedings of the 30th Canadian Medical and Biological Engineering Society Conference; 2007 Jun 16-19; Toronto, Canada.

23. Haggard P, Cockburn J. Concurrent performance of cognitive and motor tasks in neurological rehabilitation. Neuropsychol Rehabil. 1998;8(2):155-70. DOI:10.1080/713755565

24. Haggard P, Cockburn J, Cock J, Fordham C, Wade D. Interference between gait and cognitive tasks in a rehabilitating neurological population. J Neurol Neurosurg Psychiatry. 2000;69(4):479-86. [PMID: 10990508]

DOI:10.1136/jnnp.69.4.479

Submitted for publication September 1, 2010. Accepted December 2, 2010.

This article and any supplementary material should be cited as follows:

Losier Y, Englehart K, Hudgins B. Evaluation of shoulder complex motion-based input strategies for endpoint prosthetic-limb control using dual-task paradigm. J Rehabil Res Dev. 2011;48(6):669-78.

DOI:10.1682/JRRD.2010.08.0165

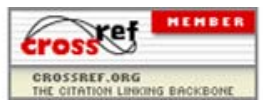

